Constitutive modeling of Radiation effects on the Permanent Set in a silicone elastomer

A. Maiti, R. Gee, T. Weisgraber, S. Chinn, R. Maxwell

March 13, 2008

Polymer Degradation and Stability 
This document was prepared as an account of work sponsored by an agency of the United States government. Neither the United States government nor Lawrence Livermore National Security, LLC, nor any of their employees makes any warranty, expressed or implied, or assumes any legal liability or responsibility for the accuracy, completeness, or usefulness of any information, apparatus, product, or process disclosed, or represents that its use would not infringe privately owned rights. Reference herein to any specific commercial product, process, or service by trade name, trademark, manufacturer, or otherwise does not necessarily constitute or imply its endorsement, recommendation, or favoring by the United States government or Lawrence Livermore National Security, LLC. The views and opinions of authors expressed herein do not necessarily state or reflect those of the United States government or Lawrence Livermore National Security, LLC, and shall not be used for advertising or product endorsement purposes. 


\title{
Constitutive modeling of Radiation effects on the Permanent Set in a silicone elastomer
}

\author{
A. Maiti, ${ }^{\dagger}$ R. H. Gee, T. Weisgraber, S. Chinn, and R. S. Maxwell
}

\begin{abstract}
When a networked polymeric composite under high stress is subjected to irradiation, the resulting chemical changes like chain scissioning and cross-link formation can lead to permanent set and altered elastic modulus. Using a commercial silicone elastomer as a specific example we show that a simple 2-stage Tobolsky model in conjunction with Fricker's stress-transfer function can quantitatively reproduce all experimental data as a function of radiation dosage and the static strain at which radiation is turned on, including permanent set, stress-strain response, and net cross-linking density.
\end{abstract}

\footnotetext{
† Corresponding author, E-mail: amaiti@1lnl.gov
} 
Filled elastomeric rubber materials are versatile components in numerous applications ranging from cushion, coating, adhesives, seismic isolation, thermal and electrical barriers and interconnects [1-3]. Such materials over long times can undergo chemical aging, which can alter and degrade many of its useful properties important to the application in question. Chemical aging signatures most profoundly affecting the elastic properties of the polymer include [4, 5]: (1) creation of new crosslinks; (2) breaking (scission) of covalent bonds; and (3) modification of the polymer-filler interface due to changes in cross-linking to the filler, as well as physico-chemical changes, e.g., involving removal of water, and so on. In applications, e.g., cushions, where the polymeric material is subjected to finite stress conditions for a long period of time, such chemical modifications can lead to a modified state of ease (i.e., a state corresponding to zero stress) known as permanent set.

Given the slow nature of the above processes one often designs artificial aging experiments in which the polymeric material is subjected to harsher, but controlled environments than it experiences in reality. A relevant experiment in this regard is a recent one performed by Chinn et al. [6], in which a commercial, filled siloxane composite, DC-745 was subjected to states of finite tensile strain $\left(\lambda_{I}\right)$ and exposed to controlled dosages $(D)$ of $\gamma$-radiation from a Co-60 source. Following the irradiation, tensile tests were performed to measure the amount of permanent set and stress-strain response for each value of $D$ and $\lambda_{l}$. In addition, NMR and swelling experiments were performed to measure the radiation-induced changes to the net cross-link density. These experiments revealed that: (1) exposure to ionizing $\gamma$-radiation primarily leads to radiative 
cross-linking reactions; and (2) the net increase in cross-linking density is proportional to the radiation dosage $D$, but nearly independent of the strain state $\lambda_{l}$ at which the radiation is applied.

More than six decades ago, a simple, yet effective model for describing permanent set under conditions described above was given by Tobolsky and his collaborators $[7,8]$. This model, an independent network hypothesis, surmises that the additional crosslinks introduced at strain $\lambda=\lambda_{I}$ can be treated as an additional independent network, whose state of ease is at strain $\lambda_{I}$. Mathematically this can be expressed by writing the total stress of the system as a sum of stress due to the original network and that due to the new network, as follows:

$$
\sigma_{\text {total }}=\sigma\left(v_{0}, \lambda\right)+\sigma\left(v_{1}, \lambda^{\prime}\right),
$$

where $\lambda$ denotes the system strain (the original zero stress corresponding to $\lambda=1$ ), $\lambda^{\prime}=\lambda / \lambda_{1}, \quad v_{0}$ is the cross-linking density of the original network, and $v_{1}$ the new crosslinking density introduced (e.g., by radiation) at $\lambda=\lambda_{1}$. The function " $\sigma$ " is the stress response function of the pristine material, for which one could potentially use one of several forms available in rubber elasticity theory [9], e.g., Neohookean, Mooney-Rivlin, Valanis-Lendel, Ogden, or more modern variants [10]. The efficacy of the Tobolsky model (eq. (1)) has been tested extensively in the literature, see, e.g., ref. [11-13].

Unfortunately, when eq. (1) in conjunction with a simple Neohookean materials model was used to interpret the permanent set data of ref. [6], the increase in crosslinking density was found to be very different from that measured in NMR and swelling 
experiments, especially at higher radiation dosage. This failure of the Tobolsky model was attributed to the creation of elastically ineffective chains through the formation of crosslinks that close off loops [6].

In this Letter, we re-investigate the above problem by using a more generalized Tobolsky model that incorporates a subtle feedback effect of the new network on the original network when new crosslink creation is also accompanied with scissioning of some of the original crosslinks [14]. Mathematically this is expressed in terms of a stress transfer function $\psi_{\text {transfer, }}$, which is the fraction of the new crosslinks that effectively become part of the original network through the feedback mechanism [15]. Thus, in the generalized Tobolsky model one replaces the crosslink densities $v_{0}$ and $v_{l}$ of eq. (1) by effective quantities:

$$
\sigma_{\text {total }}=\sigma\left(v_{0, e f f}, \lambda\right)+\sigma\left(v_{1, e f f}, \lambda^{\prime}\right)
$$

where,

$$
v_{0, e f f}=v_{0}-v_{s c i}+\psi_{\text {transfer }} v_{1} ; \text { and } v_{1, e f f}=v_{1}-\psi_{\text {transfer }} v_{1}
$$

In eq. (2) $v_{s c i}$ is the density of original cross-links that undergo scissioning at strain $\lambda_{1}$. In the following we express chain scissioning and new crosslink formation as fractions of the original crosslink density, i.e., $v_{s c i}=\xi_{s c i} v_{0}$ and $v_{1}=\xi_{x c} v_{0}$, which allows reexpressing eq. (2) as:

$$
v_{0, e f f}=v_{0}\left(1-\xi_{s c i}+\psi_{\text {transfer }} \xi_{x c}\right) ; \text { and } v_{1, e f f}=v_{0}\left(1-\psi_{\text {transfer }}\right) \xi_{x c}
$$


As for $\psi_{\text {transfer }}$ we use a simple formula developed by Fricker [16] for a system of phantom chains:

$$
\psi_{\text {transfer }}=\frac{\xi_{s c i}}{1+\xi_{x c}}
$$

which, by its very construction implies non-zero feedback only in the presence of chain scission, and has the following simple property:

$$
v_{1, e f f}=\xi_{x c} v_{0, e f f}
$$

Accuracy of the Fricker's formula (eq. (3)) for elastomeric systems undergoing simultaneous chain scission and crosslinking has been verified through explicit molecular dynamics simulations on bead-spring models [14].

In order to compute permanent set and stress-strain response, one needs: (i) a materials model for the function " $\sigma$ " of eq. (1'), and (ii) a model for $\xi_{s c i}$ and $\xi_{x c}$ as a function of the radiation dosage $D$. For simplicity, we chose the first-order MooneyRivlin materials model [9]:

$$
\sigma(v, \lambda)=v k_{B} T f(\lambda), \quad \text { where } f(\lambda)=(1+\beta / \lambda)\left(\lambda^{2}-1 / \lambda\right) /(1+\beta)
$$

In eq. (4), $\sigma$ represents the "true" stress, $k_{B}$ is the Boltzmann constant, and $\beta$ is the ratio of the Mooney-Rivlin constants $\left(\beta=C_{01} / C_{10}\right.$ in the standard notation of rubber elasticity literature [9]), which typically is in the range 0.3-1.0 for most elastomeric rubber materials. The product $v k_{B} T$ physically represents the shear modulus of the material 
under zero strain. For the radiation model, we assume a simple linear dependence of chain scission and crosslinking on dosage $D$ (within limits of $\xi_{s c i}<1$ ):

$$
\xi_{s c i}=k_{s c i} D \text {, and } \xi_{x c}=k_{x c} D
$$

In the rest of the paper we explore whether the simple theoretical model embodied in equations (1 $)-(5)$ can be used to explain the permanent set and elastic response data of ref. [6] by exploiting just four fitting variables: $v_{0}, \beta, k_{s c i}$, and $k_{x c}$.

In Fig. 1 we compare the experimental recovered length and permanent set for different values of $D$ and $\lambda_{I}$ with computed values. To compute the recovered length $\lambda_{s}$, we numerically solve eq. ( $\left.1^{\prime}\right)$ for $\sigma_{\text {total }}=0$. The permanent set $P_{\mathrm{s}}$ is simply defined by the ratio $\left(\lambda_{s^{-}}-1\right) /\left(\lambda_{1}-1\right)$. Note that when eqs. $\left(3^{\prime}\right)$ and (4) are substituted into eq. $\left(1^{\prime}\right), v_{0, \text { eff }}$ becomes a multiplicative constant, thereby yielding the following simple equation for $\lambda_{s}$ :

$$
f\left(\lambda_{s}\right)+\xi_{x c} f\left(\lambda_{s} / \lambda_{1}\right)=0,
$$

which is independent of both $v_{0}$ and (more interestingly) the amount of chain scissioning $\xi_{s c i}$ [17]. Thus, in order to compute $\lambda_{s}$, we only need values for parameters $\beta$ and $k_{x c}$, for which we made the following choices: $\beta=0.3$, and $k_{x c}=0.010$ (kGray) ${ }^{-1}$. As Fig. 1 indicates, the agreement between theory and experiment is excellent for all values, except a slight overestimation of the computed value at $D=70 \mathrm{kGray}$ and $\lambda_{I}=1.9$.

To fit the other two parameters, i.e., the initial crosslink density $v_{0}$ and the amount of radiation-induced chain scission governed by $k_{s c i}$, we needed experimental data on stressstrain response, which was available for the specific radiation dose $D=170$ kGray (see 
Fig. 5 of ref. [6]). The crosslink density $v_{0}$ could be readily obtained from the elastic response of the pristine material (i.e. not exposed to radiation), which yields $v_{0} k_{B} T=$ 135.4 psi. As for the chain scission parameter we chose $k_{s c i}=0.002$ (kGray) $)^{-1}$, which provided an excellent fit to the low-strain $(\lambda<20 \%)$ experimental data for all values of $\lambda_{1}$, as shown in Fig. 2. In particular, the observed stiffening of the material under irradiation through an increased elastic modulus is accurately reproduced in our model. It is to be noted, however, that the experimental stress-strain response for higher strains clearly deviate from the computed values. This stems from the well-known limitations of the simple first-order Mooney-Rivlin model employed here, and could be improved by using a more sophisticated materials model from the literature $[9,10]$.

Finally, we computed the net cross-link density $v_{0}+v_{x c}-v_{s c i}=v_{0}\left(1+\xi_{x c}-\xi_{s c i}\right)$ as a function of radiation dosage and compared with NMR and swelling data of ref. [6]. Fig. 3 displays such a comparison. We have also included values previously predicted from the measured permanent set data using a Neohookean materials model (see Fig. 11 of ref. [6]). Our computed cross-link density is in excellent agreement with the NMR and swelling data, clearly showing that the generalized Tobolsky model achieves remarkable consistency among all data, i.e., permanent set, stress-strain response, as well as measured cross-link density as a function of radiation dosage.

In summary, with the commercial elastomer DC-745 as a concrete example, we have demonstrated the usefulness of a generalized 2-stage Tobolsky model in conjunction with the Fricker's stress transfer function in interpreting radiation-induced chemical aging as manifested in permanent set and changes in stress-strain response. Even a simple 
materials model like first-order Mooney-Rivlin with very few parameters is able to quantitatively reproduce uniaxial tensile data over a wide range of experimental conditions, firmly establishing the strong predictive power of such an approach. Our model predicts that permanent set is independent of the amount of chain scission, while elastic response depends on both chain scission and new crosslinks created by the irradiation. Currently we are performing similar measurements on a number of elastomeric and foam systems, and adopting a finite-elements approach [18] in order to simulate more realistic geometries, stress patterns, and the effect of filler distribution morphology as well as the nature of the filler-polymer interface.

This work performed under the auspices of the U.S. Department of Energy by Lawrence Livermore National Laboratory under Contract DE-AC52-07NA27344. 
References:

1. J. E. Mark, B. Erman, and F. R. Eirich (eds), Science and Technology of Rubber, Academic Press, NY (2005).

2. C. A. Harper (ed), Handbook of Plastics, Elastomers, \& Composites, MacGraw-Hill, New York (2002).

3. A. Ciesielski, An Introduction to Rubber Technology, Rapra Technology Ltd., UK (1999).

4. A. V. Tobolsky, Properties and Structure of Polymers, Wiley, New York (1960).

5. J. G. Curro and E. A. Salazar, J. App. Polym. Sci. 19, 2571 (1975).

6. S. Chinn, S. DeTeresa, A. Sawvel, A. Shields, B. Balazs, and R. S. Maxwell, Polym. Degrad. Stab. 91, 555 (2006).

7. A. V. Tobolsky, I. B. Prettyman, and J. H. Dillon, J. App. Phys. 15, 380 (1944).

8. R. D. Andrews, A. V. Tobolsky, and E. E. Hanson, J. App. Phys. 17, 352 (1946).

9. L. R. G. Treloar, The Physics of Rubber Elasticity, Clarendon Press, Oxford (1975).

10. J. S. Bergström and M. C. Boyce, J. Mech. Phys. Solids 46, 931 (1998).

11. J. A. Shaw, A. S. Jones, and A. S. Wineman, J. Mech. Phys. Solid. 53, 2758 (2005).

12. P. H. Mott and C. M. Roland, Macromolecules 33, 4132 (2000). 
13. P. G. Santangelo and C. M. Roland, Rubber Chem. Technol. 76, 892 (2003).

14. D. R. Rottach, J. G. Curro, J. Budzien, G. S. Grest, C. Svaneborg, and R. Everaers, Macromolecules 40, 131 (2007).

15. The concept of stress transfer function was first introduced by Flory, where he derived an expression for $\psi_{\text {transfer }}$ for a system of Gaussian chains. See, P. J. Flory, Trans. Faraday Soc. 56, 722 (1960). We use a much simpler form due to Fricker [16].

16. H. S. Fricker, Proc. R. Soc. London A 335, 269 (1973).

17. The special case $\beta=0$ corresponds to the Neohookean model. For this case one can solve eq. (6) analytically and obtain a closed-form analytical solution for the recovered length: $\lambda_{s}=\left\{\frac{1+\xi_{x c} \lambda_{1}}{1+\xi_{x c} / \lambda_{1}^{2}}\right\}^{1 / 3}$.

18. See articles in: Constitutive Models for Rubber V, Proceedings of the $5^{\text {th }}$ European Conference, A. Boukamel, L. Laiarinandrasana, and S. Meo (eds), CRC Press (2007). 
Figure captions:

Fig. 1. Experimental (from ref. [6]) and computed recovered length $\left(\lambda_{s}\right)$ and permanent set $P_{s}\left(=\left(\lambda_{s}-1\right) /\left(\lambda_{l}-1\right)\right)$ for various values of $\lambda_{l}$ and radiation dosage $D$. (Top left) Experimental $\lambda_{s}$; (top right) computed $\lambda_{s}$; (bottom left) experimental $P_{s}$; (bottom right) computed $P_{s}$. Parameters used: $\beta=0.3, k_{x c}=0.010(\mathrm{kGray})^{-1}$.

Fig. 2. Stress-strain response data at a radiation dosage of $D=170 \mathrm{kGray}$. Response of the pristine material is included as well. (Left) Experimental (from ref. [6]) data; (Right) computed values. Parameters used: $\beta=0.3, k_{x c}=0.010(\mathrm{kGray})^{-1} ; k_{s c i}=$ $0.002(\mathrm{kGray})^{-1} ; \quad v_{0} k_{B} T=135.4$ psi. In both plots the $y$-axis represents the engineering stress, i.e., $\sigma_{e}=\sigma / \lambda$, and all curves are shifted along the negative $x$ axis by $\varepsilon_{s}=\lambda_{s^{-}} 1$ (so that they start from the origin).

Fig. 3. Net crosslink density (expressed as a fraction of the initial crosslink density $v_{0}$ ) as a function of radiation dosage. 

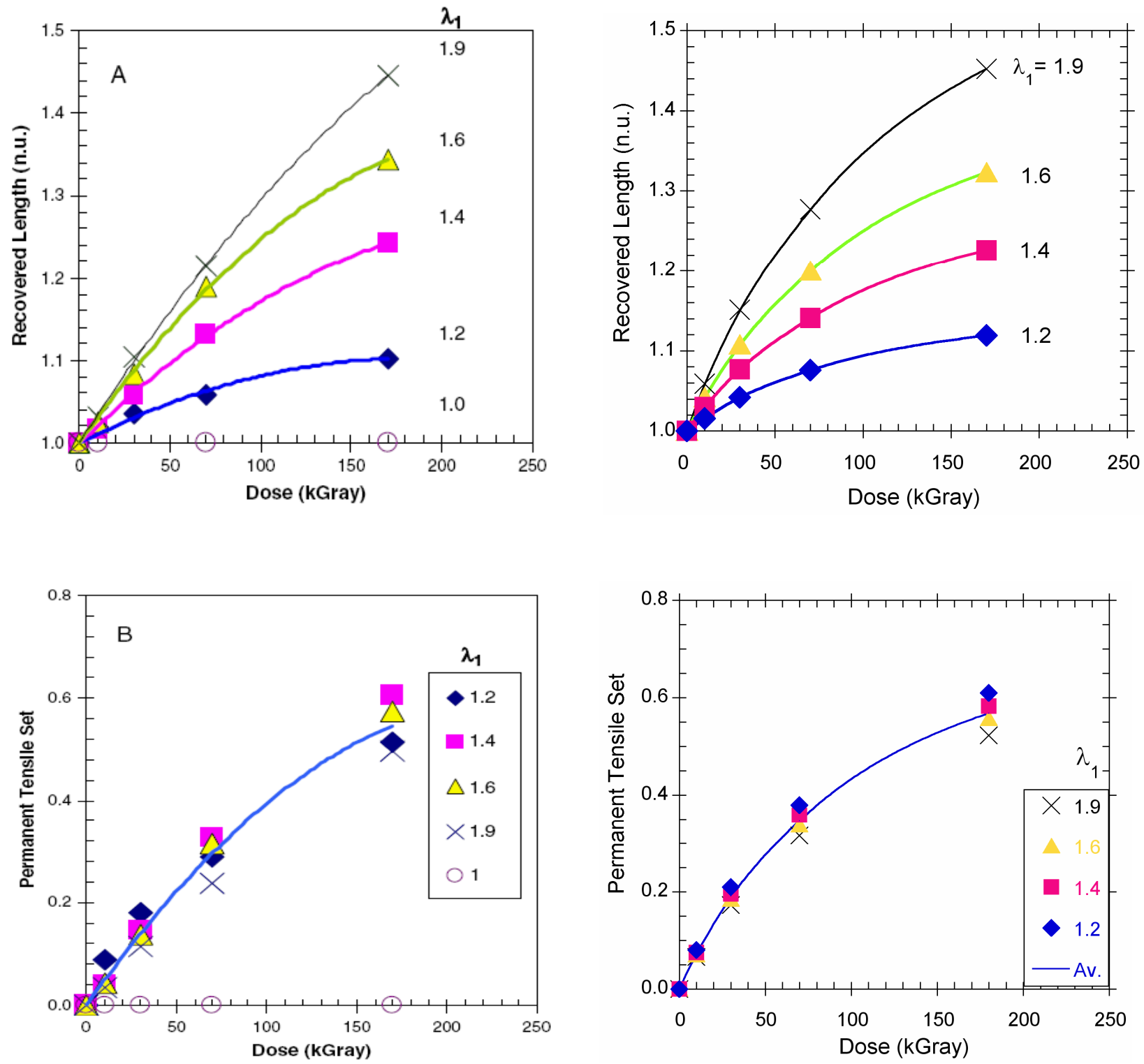

Figure 1 

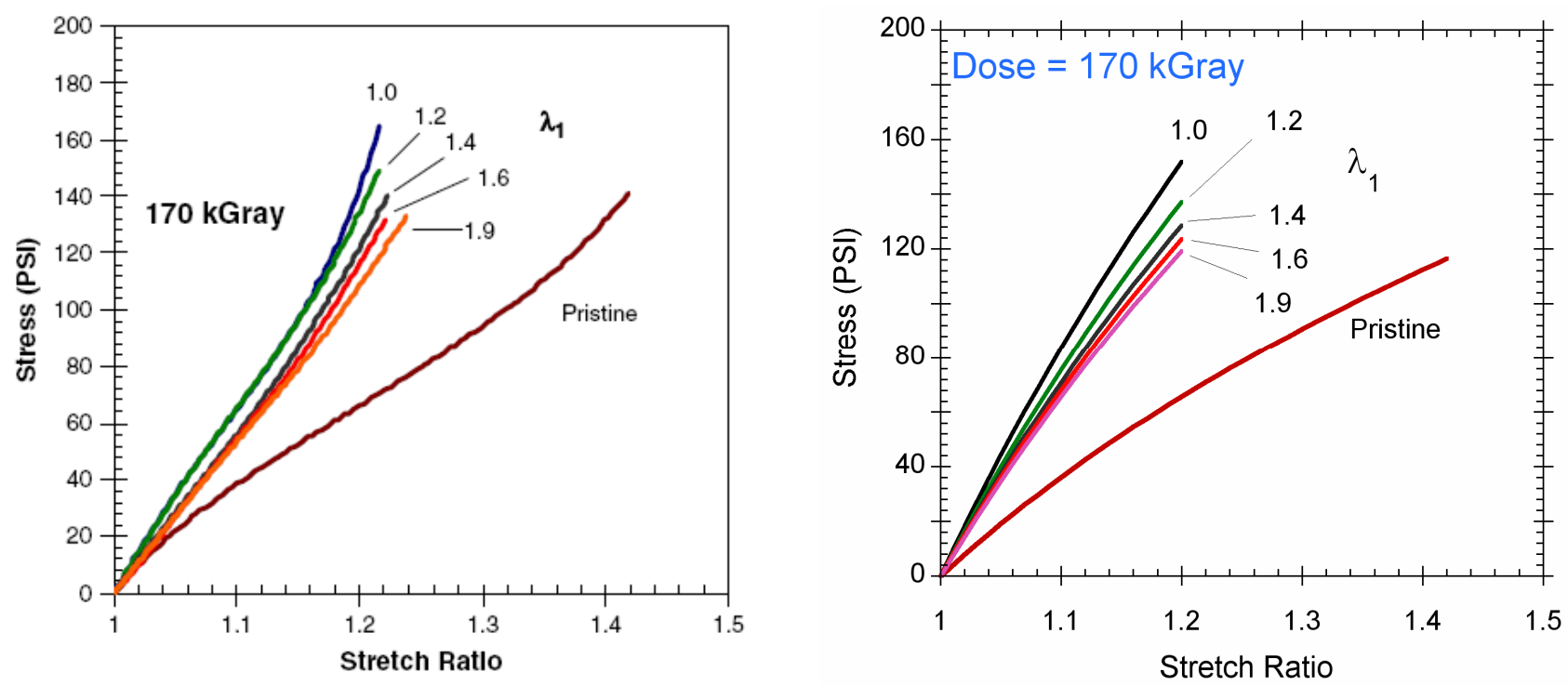

Figure 2 


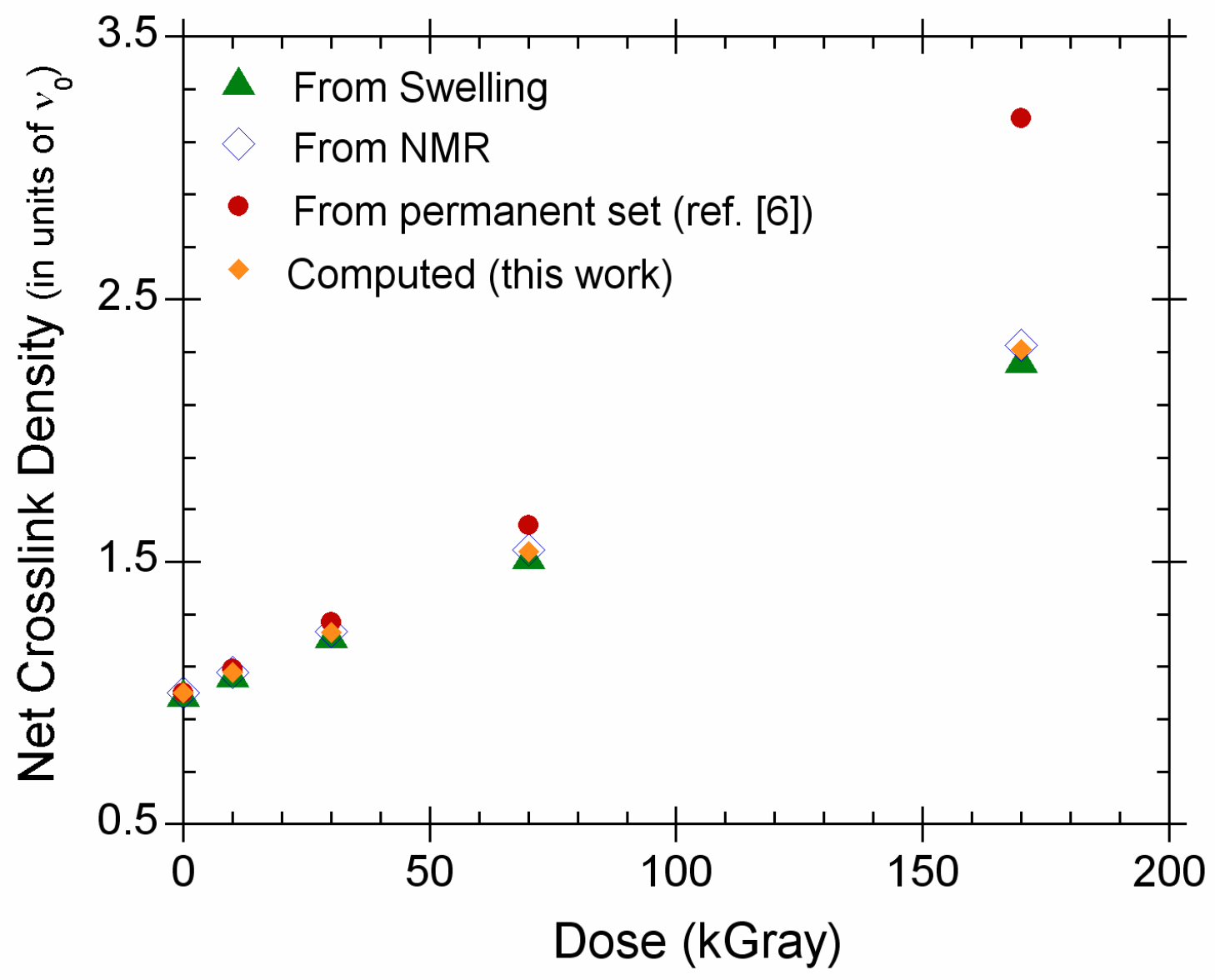

Figure 3 